\title{
Effectiveness of planned teaching programme on knowledge regarding prevention and management of thrombophlebitis in cancer patients receiving chemotherapy among staff nurses
}

\author{
Binu Babu ${ }^{1}$, Jincy Ealias ${ }^{2}$ \\ ${ }^{I}$ (Ph.D. Scholar, Nursing Department, JJT University, Rajasthan, India) \\ ${ }^{2}$ (Ph.D. Scholar, Nursing Department, JJT University, Rajasthan, India)
}

\begin{abstract}
As chemotherapy administration is the responsibility of the nursing personnel, it has been a major concern of nurses to prevent any damage to the veins resulting from thrombophlebitis.

Objectives: The objective of the study was to determine the effectiveness of planned teaching programme on knowledge regarding prevention and management of thrombophlebitis in cancer patients receiving chemotherapy among staff nurses.
\end{abstract}

Method: Quasi experimental design was used to assess the effectiveness of planned teaching programme among staff nurses. 40 staff nurses were selected from selected oncology hospitals using convenient sampling technique. The data were collected with demographic proforma and structured knowledge questionnaire.

Result: Analysis was done by descriptive and inferential statistics. Paired t-test was used to compute effectiveness of planned teaching programme. The result showed that in post test $67.5 \%$ staff nurses gained average knowledge and 32.5\% staff nurses gained good knowledge regarding prevention and management of thrombophlebitis in cancer patients receiving chemotherapy. The mean pre-test score of knowledge increased from 12.45 to 22.05 in post test.

Conclusion: The study drawn to the conclusion that the planned teaching programme was effective in improving the knowledge of staff nurses regarding prevention and management of thrombophlebitis in cancer patients receiving chemotherapy.

Keywords: Chemotherapy, Prevention and management of thrombophlebitis, Staff nurses, Thrombophlebitis.

\section{Introduction}

Cancer is a disease which alters the normal mechanism of the control of growth and proliferation of a cell. It spreads directly to surrounding tissue and to new body organs other than the primary site. The incidence of cancer among the Indian population as per the ICMR in the year 2010 is a total 979,787 cases.[1] Major treatment modalities of cancer are chemotherapy, radiation, immunotherapy and surgery. The choice of particular cancer treatment depends on the stage of cancerous tumor. As per the 2010 statistics about 6 lakh patients all over the country undergo chemotherapy.

Chemotherapy, which is a common treatment modality, uses antineoplastic agents in an attempt to destroy tumor cells by interfering cellular functions and multiplication. Chemotherapeutic drugs are known to be the most irritant and a vesicant drug as it has to act on the most deadly, rapidly multiplying cells of cancer. Most chemotherapeutic agents cause nausea, vomiting, diarrhea, anemia and myelosuppression. Myelosuppression decreases the number of white and red blood cells and platelets and increases the risk for infection and bleeding. Thus increases the chance of thrombophlebitis. Thrombophlebitis is a complication that is commonly associated with intra venous therapy. Thrombophlebitis is a swelling or inflammation of a vein which can be caused by a blood clot. The symptoms often associated with thrombophlebitis are inflammation of the part affected, pain, skin redness and warmth.[2]

Chemotherapeutic drugs are infused in vein using peripheral or central catheters. Because of the powerful effect of the chemotherapeutic drugs it cannot be administered through a peripheral intravenous route for a long period of time. So it becomes mandatory to use the available venous access carefully for administering chemotherapeutic drugs. Thus the common complications such as thrombophlebitis and extravasation can be prevented and thereby preventing the permanent damage to the veins.[2] A number of literature articles have written that phlebitis can occur in as much as $25-70 \%$ of patients.[3]

Nurses are the ones who administer chemotherapy to cancer patients in the ward, so it is necessary that nurses should have adequate knowledge about thrombophlebitis, its prevention and management. 


\section{Problem Statement}

"A study to assess the effectiveness of planned teaching programme on knowledge regarding prevention and management of thrombophlebitis in cancer patients receiving chemotherapy among staff nurses working at selected oncology hospitals".

\subsection{Objectives}

The objectives of the study are;

- To assess the existing knowledge among staff nurses regarding prevention and management of thrombophlebitis in patients receiving chemotherapy.

- To determine the effectiveness of planned teaching programme on the knowledge of staff nurses regarding prevention and management of thrombophlebitis in patients receiving chemotherapy.

- To find the association between knowledge among staff nurses regarding prevention and management of thrombophlebitis in patients receiving chemotherapy with selected socio demographic variables.

\subsection{Hypothesis}

Ho: There will be no significant difference between pre and post test score.

$\mathrm{H}_{1}$ : There will be significant difference between pre-test and post test score.

$\mathrm{H}_{2}$ : There will be significant association between pretest knowledge score with selected demographic variables.

\section{Review of Literature}

A study was conducted on 72 outpatients receiving anticancer therapy through an administration site distal to previous vein puncture in the same arm in a tertiary cancer center. Participants were assessed before treatment and on $7^{\text {th }}$ day for incidence of extravasation/phlebitis. This study aims to identify that the practice of 24-hour interval between a venous puncture and subsequent cannulation at a distal site is necessary for preventing extravasation. The study concluded that a nurse can administer anticancer therapy at a distal site if the vein of choice is not connected to the previous puncture site.[4]

A retrospective analysis was conducted on the advantage of peripherally inserted central venous catheters over conventional central venous catheter in Rajiv Gandhi Cancer Institute and Research Centre, Delhi among children with cancer with regard to the demographic data, catheter life, reason for removal, and complications. The study concluded that the common causes for catheter removal were suspected infection, dislodgement, phlebitis, and occlusion with the rates of $1.27,0.31,0.06$, and 0.06 per 1,000 catheter days, respectively.[5]

A study was conducted to examine the impact of implementation of guidelines for the management of peripheral intravascular devices on nurses' knowledge and practice as well as patient outcome in a Hong Kong tertiary care teaching hospital. The results showed that there is decrease in the incidence of extravasations and phlebitis with significant improvement in practice. Thus concluded that the implementation of guidelines served as an important measure for the prevention of complications related to intravascular devices.[6]

A prospective study conducted to compare knowledge on principles with practice of vein care during intra venous therapy at a teaching hospital. This evaluated the effect of an educational program on the knowledge and practice of persons responsible for vein care. The study concluded that careful supervision may lead to improved vein care and a reduction in infusion related phlebitis.[7]

\subsection{Design and sampling}

\section{Methodology}

An evaluative study was conducted using quasi experimental design to assess the effectiveness of planned teaching programme on knowledge regarding prevention and management of thrombophlebitis in cancer patients receiving chemotherapy among staff nurses. Target population is staff nurses. Convenient sampling technique is used for selecting 40 staff nurses working in selected oncology hospitals and caring for patients undergoing chemotherapy.

\subsection{Instrument}

In this study, the tool for data collection includes the demographic proforma and the structured knowledge questionnaire to assess the knowledge of staff nurses on prevention and management of thrombophlebitis in cancer patients receiving chemotherapy. The demographic proforma consist of questions on demographic variables like age, gender, professional qualification, experience, and experience in oncology unit, and previous information regarding prevention and management of thrombophlebitis. The structured knowledge questionnaire includes 7 items for knowledge regarding cancer and chemotherapy, 9 items for knowledge regarding thrombophlebitis and 14 items regarding prevention and management of thrombophlebitis. Each item has 4 options with one correct answer. A score of ' 1 ' ' is awarded for the correct answer and a score of ' 0 ' is 
awarded for the wrong answer. Total score is 30 . The knowledge level has been arbitrarily divided into three categories based on the scores in the structured questionnaire.
- Poor knowledge
: (scores $1-10)$
- Average knowledge
: (scores $11-20)$
- Good knowledge
: (scores $21-30)$

The tool prepared was validated by the experts. The reliability of the tool was done using split- half method on 10 staff nurses and it was found to be highly significant, $r=0.823$.

A planned teaching programme on prevention and management of thrombophlebitis in cancer patients receiving chemotherapy is prepared with the objective to improve the knowledge among staff nurses working in selected oncology hospitals. A 45 minute lecture cum discussion class is planned using AV aids such as power point, flash cards and OHP sheets. The section discussed the meaning, incidence and treatment modalities of cancer, procedures used for chemotherapy, meaning, etiology, signs and symptoms, investigations, complications, preventive measures and management of thrombophlebitis including medical, surgical and nursing.

\subsection{Data collection procedure}

Prior permission is obtained from the respective authorities. The purpose of the study, method of data collection and time duration were explained to the subjects for getting free true responses. They were also given assurance regarding the confidentiality of the information. An informed consent was obtained from the respondents indicating their willingness to participate in the study. Subjects who fulfilled the sampling criteria were taken from the selected oncology hospitals. The main study was conducted on three phases. In phase 1, pre-test was done using the demographic proforma and the structured knowledge questionnaire. In phase 2 , planned teaching was done on the 2 nd day of the pre-test. And in phase 3, post test was conducted on the 8th day using the same knowledge questionnaire on same samples who responded to pre test and attended the planned teaching programme. The data collected in the phase 1 and 3 were analyzed using descriptive and inferential statistics and interpreted in terms of the objectives and hypothesis of the study.

\section{Findings of the Study}

The collected data from 40 staff nurses organized and analyzed in terms of objectives and hypothesis. The demographic data is analyzed in terms of frequency and percentage. The data shows majority of samples $29(72.5 \%)$ belong to age between $21-25$ years, 33 samples $(82.5 \%)$ are females, 35 (87.5\%) have GNM qualification, 18 nurses (45\%) have total experience between 1-2 years, 32 nurses $(80 \%)$ have less than 1 year experience in oncology wards and 37 nurses $(92.5 \%)$ has not attended any workshop or seminar on prevention and management of thrombophlebitis.

The pretest knowledge score distribution of staff nurses regarding prevention and management of thrombophlebitis in cancer patients receiving chemotherapy is shown below.

Table 1: Distribution of staff nurses according to pretest knowledge score $(\mathrm{N}=40)$

\begin{tabular}{|l|c|c|}
\hline \multicolumn{1}{|c|}{ Level of knowledge score } & Frequency & Percentage (\%) \\
\hline Poor knowledge & 18 & 45 \\
\hline Average knowledge & 18 & 45 \\
\hline Good knowledge & 4 & 10 \\
\hline
\end{tabular}

TABLE 1 indicates that 18 respondents belong to poor knowledge category, 18 belong to average knowledge category and 4 samples belong to good knowledge category. Area wise distribution of knowledge in pretest knowledge score in terms of mean and standard deviation also identified and is as shown below.

Table 2: Area wise distribution of knowledge in pretest knowledge score $(\mathrm{N}=40)$

\begin{tabular}{|c|l|c|c|c|}
\hline Sl.no & \multicolumn{1}{|c|}{ Knowledge area } & Maximum score & Mean & SD \\
\hline 1. & Cancer and chemotherapy & 7 & 3.35 & 1.647 \\
\hline 2. & Thrombophlebitis & 9 & 3.675 & 1.992 \\
\hline 3. & $\begin{array}{l}\text { Prevention and management of } \\
\text { thrombophlebitis }\end{array}$ & 14 & 5.425 & 2.286 \\
\hline
\end{tabular}

TABLE 2 highlights that the samples were having less knowledge regarding prevention and management of thrombophlebitis (mean $=5.425$ ) and the knowledge about cancer and chemotherapy has highest mean of 3.35, the mean knowledge score of staff nurses about thrombophlebitis was 3.675.

The post test knowledge score distribution of staff nurses regarding prevention and management of thrombophlebitis in cancer patients receiving chemotherapy is presented below. 
Table 3: Distribution of staff nurses according to post test knowledge score $(\mathrm{N}=40)$

\begin{tabular}{|l|c|c|}
\hline \multicolumn{1}{|c|}{ Level of knowledge score } & Frequency & Percentage (\%) \\
\hline Poor knowledge & 0 & 0 \\
\hline Average knowledge & 13 & 32.5 \\
\hline Good knowledge & 27 & 67.5 \\
\hline
\end{tabular}

TABLE 3 specifies that majority $67.5 \%$ of the staff nurses have good knowledge and $32.5 \%$ staff nurses have average knowledge regarding prevention and management of thrombophlebitis in cancer patients receiving chemotherapy.

Paired t-test is used for analyzing the effectiveness of planned teaching programme on knowledge regarding prevention and management of thrombophlebitis in cancer patients receiving chemotherapy among staff nurses. The paired t-test value is 14.14 and is tested at $p<0.05$ level of significance. The mean pre-test score of knowledge increased from 12.45 to 22.05 in post test. As there is significant difference in pretest and post-test knowledge score, the hypothesis $\mathrm{H}_{0}$ is rejected and $\mathrm{H}_{1}$ is accepted. Thus the planned teaching is statistically significantly effective in improving knowledge of staff nurses regarding prevention and management of thrombophlebitis in patients receiving chemotherapy.

The association between pretest knowledge score and the socio-demographic variables were computed using the chi- square test. There was no significant association established between demographic variables with pre-test knowledge regarding prevention and management of thrombophlebitis in patients receiving chemotherapy. Therefore hypothesis $\mathrm{H}_{2}$ is rejected.

\section{Conclusion}

Prevention of thrombophlebitis in chemotherapy administration or at least bringing down the alarmingly rates of thrombophlebitis is very important for reducing the progression of disease especially in cancer patients. Nurses are the one who provide front line care to chemotherapy patients playing various roles from the admission, insertion of peripheral line till the finish of chemotherapy. Thus it is vital to educate the nurses about thrombophlebitis and its complications and how to do ongoing assessment for the early symptom detection of thrombophlebitis. The present study conducted to assess the effectiveness of planned teaching programme on knowledge regarding prevention and management of thrombophlebitis in cancer patients receiving chemotherapy among staff nurses working at selected oncology hospitals. The study result highlights that majority of staff nurses upgraded their knowledge regarding prevention and management of thrombophlebitis in cancer patients receiving chemotherapy after the administration of planned teaching programme. The study drawn to the conclusion that planned teaching programme was effective in improving the knowledge of staff nurses regarding prevention and management of thrombophlebitis in cancer patients receiving chemotherapy.

The study findings can be implicated in nursing education and practice. Nursing curriculum should incorporate a section on educating prevention and management of thrombophlebitis, improving the skills of monitoring intravenous lines. Further research in this area can be undertaken in different fields to quantify the magnitude of deficiency of knowledge prevention and management of thrombophlebitis and the needful can be done.

\section{References}

[1] J. Ferlay, H.R. Shin, F. Bray, D. Forman, C. Mathers and D.M. Parkin, Estimates of worldwide burden of cancer in 2008: GLOBOCAN 2008, International Journal of Cancer, 127(12), 2010, 2893-2917.

[2] S.C. Smeltzer, Brunner and Siddarth textbook of medical surgical nursing (Lippincot Publication, New York, 2010).

[3] P. Wallis, Phlebitis, Intravenous nursing, Newsletter, New Zealand, 2003-2006.

[4] R.J. Chan, A. Alexander, M. Bransdon, J. Webster, B.G. Hughes, L. Brown, T. Graham et. al, Challenging the distal-to-proximal cannulation technique for administration of anticancer therapies, Cancer nursing, 2011.

[5] S. Abedin, G. Kapoor, Peripherally inserted central venous catheters are a good option for prolonged venous access in children with cancer, Pediatric Blood \& Cancer, 51(2), 2008, 251-255.

[6] V. Lopez, A. Molassiotis and W.K. Chan, Evaluate Nursing Management of Peripheral Intravascular Devices, Journal of Infusion Nursing, 27(5), 2004, $322-331$.

[7] B.J. Brown, P.A. Mackowiak and J.W. Smith, Care of veins during intravenous therapy, American Journal of Infection Control, $8(4), 1980,107-112$. 